\title{
La economía de Filipinas
}

$\mathrm{E}$ 1 archipiélago filipino, compuesto por más de 7000 islas, tiene un tamaño similar al de Japón y representa alrededor de una quinta parte del territorio mexicano. El archipiélago se distribuye en forma de triángulo: la isla de Palawan constituye uno de sus lados y las islas de Sulu y la de Mindanao forman la base en el sur, mientras que las islas Batan en el norte constituyen el vértice. En las planicies de la isla de Luzón, situada al norte, y de la isla Panay al centro se concentra la mayor parte de la población (ver mapa). En Luzón se encuentra la capital, Manila, cuya área metropolitana tiene una densidad de población de las más altas del mundo, 11000 habitantes por kilómetro cuadrado, la cual es similar a la de Tokio.

Con una población de 76 millones, Filipinas es el cuarto país más poblado en el que se habla inglés; además, el único en el sudeste asiático mayoritariamente católico. Lo anterior es un reflejo de la influencia occidental de cuatro siglos: bajo el dominio español (1521 a 1898), y de ahí hasta la década de los cuarenta bajo el dominio norteamericano. Durante la Segunda Guerra permaneció bajo el control nipón por cuatro años (1942-1945); los efectos fueron devastadores en términos de pérdida de vidas y de la destrucción material, sobre todo en el área de Manila, la capital.

Sin embargo, a partir de la independencia de Filipinas, en 1946, el futuro del país lucía promisorio. Durante los primeros años de independencia, el país se recuperaba con la asistencia de Estados Unidos. Rica en recursos minerales como hierro, cobre, plomo y zinc, y en recursos forestales y agrícolas (además de contar con 33600 kilómetros de costas), Filipinas contaba con la dotación de recursos necesarios para desarrollarse. Se basaría en la construcción

* Investigadora del Departamento de Estudios del Pacífico de la Universidad de Guadalajara.

ORCID http://orcid.org/0000-0003-4926-0594 de la identidad asiática hecha a un lado durante los siglos de dominio occidental. Así, Filipinas se perfilaba como la nación que llevaría la delantera, en el sendero del desarrollo, con respecto a sus vecinos del sudeste asiático. Paradójicamente, como lo señalan Simone y Thompson, Filipinas, junto con Myanmar, han constituido "las excepciones de la historia de éxito de Asia Pacífico". ${ }^{1}$ La inestabilidad política, el descontento social manifestado en varias insurrecciones armadas, el excesivo endeudamiento externo y el deterioro de las finanzas públicas han sido algunos de los factores más sobresalientes que han retardado el desarrollo económico de Filipinas.

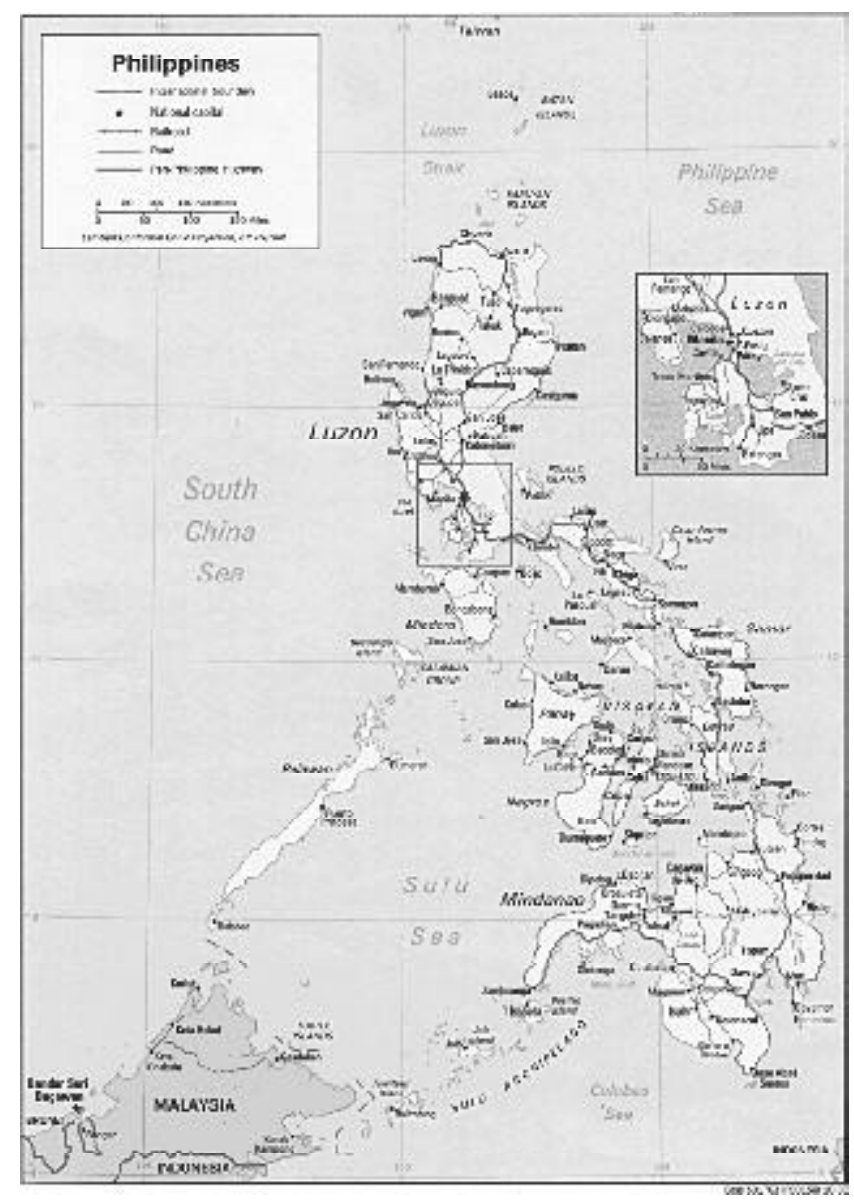




\section{Situación actual}

Por su tamaño, la economía de Filipinas representa una cuarta parte de la de México. Los filipinos poseen un ingreso per cápita de 3670 dólares internacionales (medida que considera el poder de compra del ingreso), los cuales representan menos de la mitad del ingreso que perciben los mexicanos (cuadro 2); ello no obstante que durante los noventa (19901997), la economía filipina tuvo un desempeño mejor que el de la mexicana, con una tasa de crecimiento promedio muy superior a la de México.

La estructura productiva del país se ha modificado lentamente en las últimas tres décadas. En 1970 el producto interno bruto (PIB) agrícola representaba 30 por ciento de la producción total. Actualmente, la economía de Filipinas es todavía muy dependiente de ese sector. Alrededor de una quinta parte del PIB filipino se genera en la agricultura, la cual emplea cerca de 40 por ciento de la población económicamente activa, reflejando una baja productividad agrícola. Esta última se ha visto afectada por una infraestructura inadecuada, falta de financiamiento y una reforma agraria inconclusa. Entre los principales productos agrícolas de Filipinas figuran el azúcar, los productos de coco, el arroz, el maíz, las piñas, los plátanos, mangos, cerdo y huevo.

El sector industrial, por su parte, ha mantenido una participación en el PIB de alrededor de 30 por ciento, con predominio de la industria ligera y de industrias basadas en recursos naturales. El sector industrial está concentrado en las áreas urbanas, especialmente en el área metropolitana de Manila. $\mathrm{Su}$ desarrollo se ha visto limitado por la falta de infraestructura, de comunicaciones y de transporte, así como por la escasa generación de energía eléctrica. Entre los principales sectores industriales se encuentran el de textiles y ropa; el de farmacéuticos, el de químicos, el de productos de madera, el de alimentos, el ensamble de productos electrónicos y la refinación de petróleo.

\section{Evolución de la economía}

A partir de los sesenta Filipinas aplicó un modelo de sustitución de importaciones que protegía a su industria incipiente. El modelo parecía dar resultados. En la década de los setenta la economía crecía a una tasa promedio de 6 por ciento, con una inflación promedio anual de 13.4 por ciento. Sin embargo, el reducido tamaño del mercado local impidió el ulterior desarrollo de la industria y ya para fines de los setenta, aquel impuso un límite al modelo de desarrollo cerrado con sustitución de importaciones. Sin embargo, los poderosos intereses de los grupos industriales, fuertemente vinculados a la oligarquía terrateniente, impidieron el cambio hacia el modelo aplicado en las otras economías asiáticas, el cual se orientaba a la promoción de las exportaciones.
La elevación de los precios del petróleo, tanto a mediados como a fines de los setenta, y de cuyas importaciones Filipinas es altamente dependiente, tuvo un impacto muy negativo sobre la economía. Así, a partir de 1981 la economía empezó a desacelerar su crecimiento; en 1984 y 1985 ésta presentó una severa contracción de 7 por ciento en cada año. La anterior situación se vio agravada por el excesivo 
endeudamiento externo en que había incurrido el gobierno de Ferdinand Marcos. La deuda externa del sector público pasó de representar 3 por ciento del PIB, al inicio del segundo período de gobierno de Marcos (1969), a constituir 26 por ciento del PIB en 1984. Lo anterior tuvo como resultado una elevada carga del servicio de la deuda externa, que llegó a representar en 1986 dos terceras partes de los ingresos por exportaciones. Durante todo ese período, las finanzas públicas arrojaron saldos deficitarios.

El incremento de los precios del petróleo, el proteccionismo que impedía la entrada de productos más baratos y las devaluaciones de principios de los setenta y de mediados de los ochenta, mantuvieron la inflación en los dos dígitos durante todo el período 1969-1985, la cual se aceleró durante la primera mitad de los ochenta a un promedio de 20 por ciento anual. Lo anterior, aunado a una tasa de crecimiento poblacional muy alta (2.3 por ciento promedio anual), dio como resultado que en la primera mitad de los ochenta el ingreso per cápita de los filipinos tuviera un deterioro anual de 2.6 por ciento (cuadro 1 ).

Durante la segunda mitad de los ochenta la economía retomó el crecimiento. Fue en el gobierno de Corazón Aquino (1986-1991) cuando se inició un cambio de política económica hacia una economía más abierta. Durante ese período las exportaciones, incluyendo los servicios, pasaron de representar 28 por ciento del PIB a 34 por ciento. La inflación se redujo y las devaluaciones del peso fueron de magnitud menor a las de 1984 y 1985. Sin embargo, el déficit fiscal siguió creciendo y, con éste, la deuda externa pública. Durante el gobierno de Aquino se adoptó la nueva constitución de 1987 bajo el modelo norteamericano. En la constitución se reconocieron las regiones autónomas musulmanas de Mindanao y la Cordillera en la región norte de Luzón.

En el siguiente período, el gobierno de Ramos (1992-1997) continuó con la política de mayor apertura, liberalizando las telecomunicaciones, desregulando los sistemas de transporte aéreo y marítimo y privatizando muchas empresas controladas por el sector público, incluyendo la distribución de los servicios del agua en Manila. En el plano político, Ramos estableció como prioridad la "reconciliación nacional" con los comunistas, los insurgentes, los separatistas musulmanes y los rebeldes militares. La economía progresó a tasas positivas y crecientes en todo el período. En los últimos cuatro años el ingreso per cápita tuvo una recuperación sostenida y la inflación se mantuvo a un promedio de 8 por ciento; en tanto el déficit del gobierno se convirtió en superávit impulsado por el crecimiento económico, el programa de privatización y los controles al gasto del gobierno. Las exportaciones pasaron a representar 50 por ciento del PIB (cuadro 1).

En ambos períodos (1986-1997), la industria mostró tasas de crecimiento positivas, de 3.3 por ciento promedio anual, superior a las mostradas por el sector agrícola, de 1.8 por ciento. El mayor crecimiento del sector manufacturero se vio reflejado en la estructura de las exportaciones. Las exportaciones manufactureras pasaron de 16 por ciento de las exportaciones totales en 1976 y 30 por ciento en 1986, a 83 por ciento de las ventas al exterior en 1996. En esos últimos diez años, las exportaciones de manufacturas se multiplicaron por once veces, al pasar de un nivel de 1458 a 17006 millones de dólares. Sin embargo, cabe hacer notar que muchos de los bienes exportados tienen un contenido de importación muy alto. Así, durante el período mencionado las compras de manufacturas al exterior también se multiplicaron por once veces. En este sentido, la situación de Filipinas se asemeja a la del sector exportador de maquila mexicano, con su elevada dependencia de insumos importados, lo que refleja el bajo grado de integración de las empresas exportadoras con la pequeña y mediana empresa de ambos países.

Para 1998, en el gobierno de Estrada, la economía volvió a sufrir una contracción ocasionada por el fenómeno climático El Niño y algunos tifones que afectaron la producción agrícola del archipiélago. Para 1999 la economía se recuperaba de esos efectos adversos. Por otra parte, la crisis de Asia no tuvo un impacto tan 
Cuadro 1

Filipinas: evolución de principales indicadores económicos

(1969-1999)

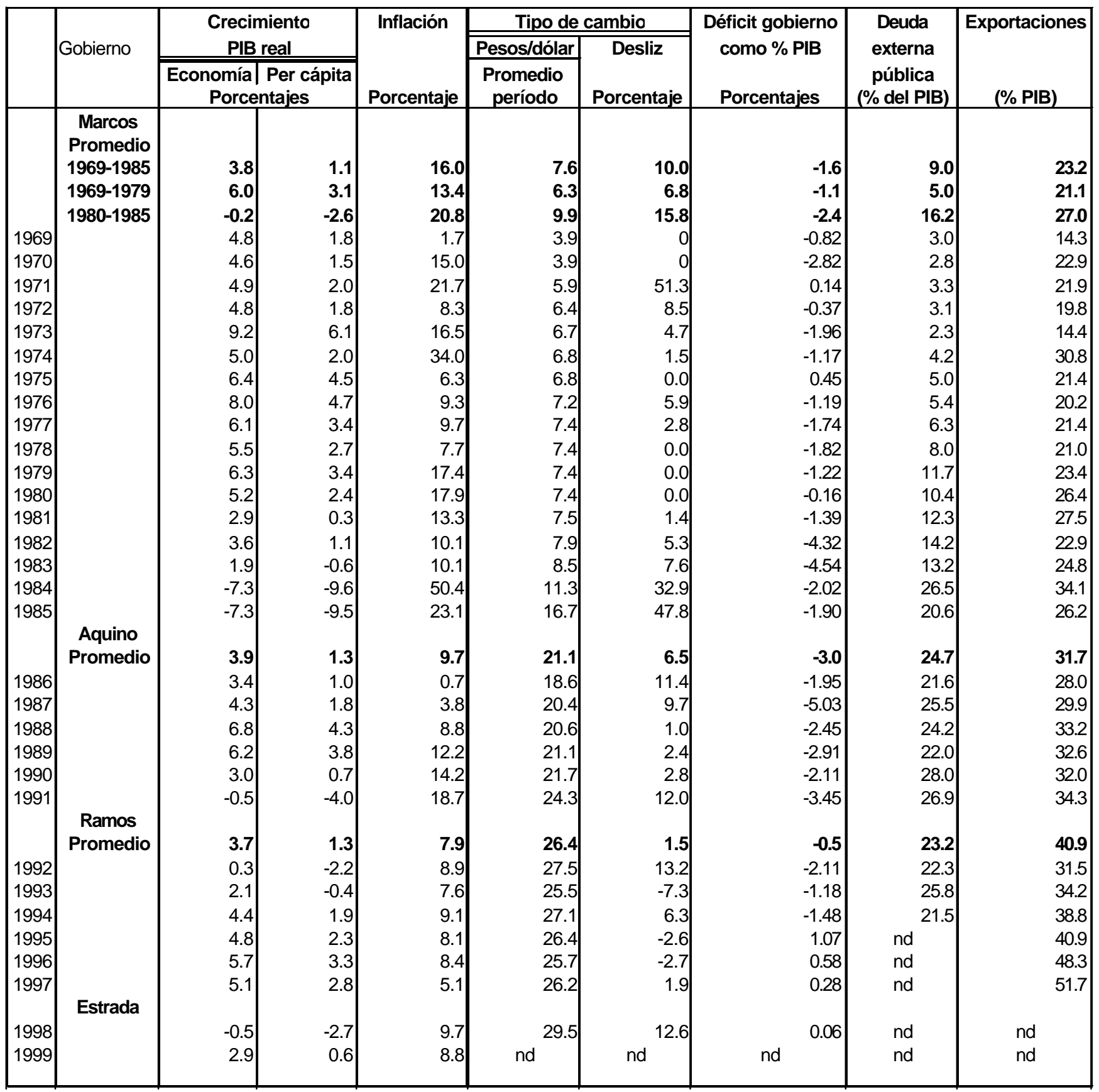

Fuente: Fondo Monetario Internacional, Estadísticas Financieras Internacionales, Anuario 1998.

nd=no disponible

devastador sobre la economía filipina, en parte porque su dependencia de financiamiento de capital de corto plazo era mucho menor que la de sus vecinos.
El gobierno de Estrada ha continuado con los programas de liberalización de sus dos inmediatos antecesores. En 1998 se eliminó la regulación sobre los productos del petróleo "socialmente sensibles" como el kerosene, gas y gasolina. Debido a las restricciones presupuestales, el gobierno se ha visto en la necesidad de 
Cuadro 2

Filipinas y México: indicadores básicos 1997

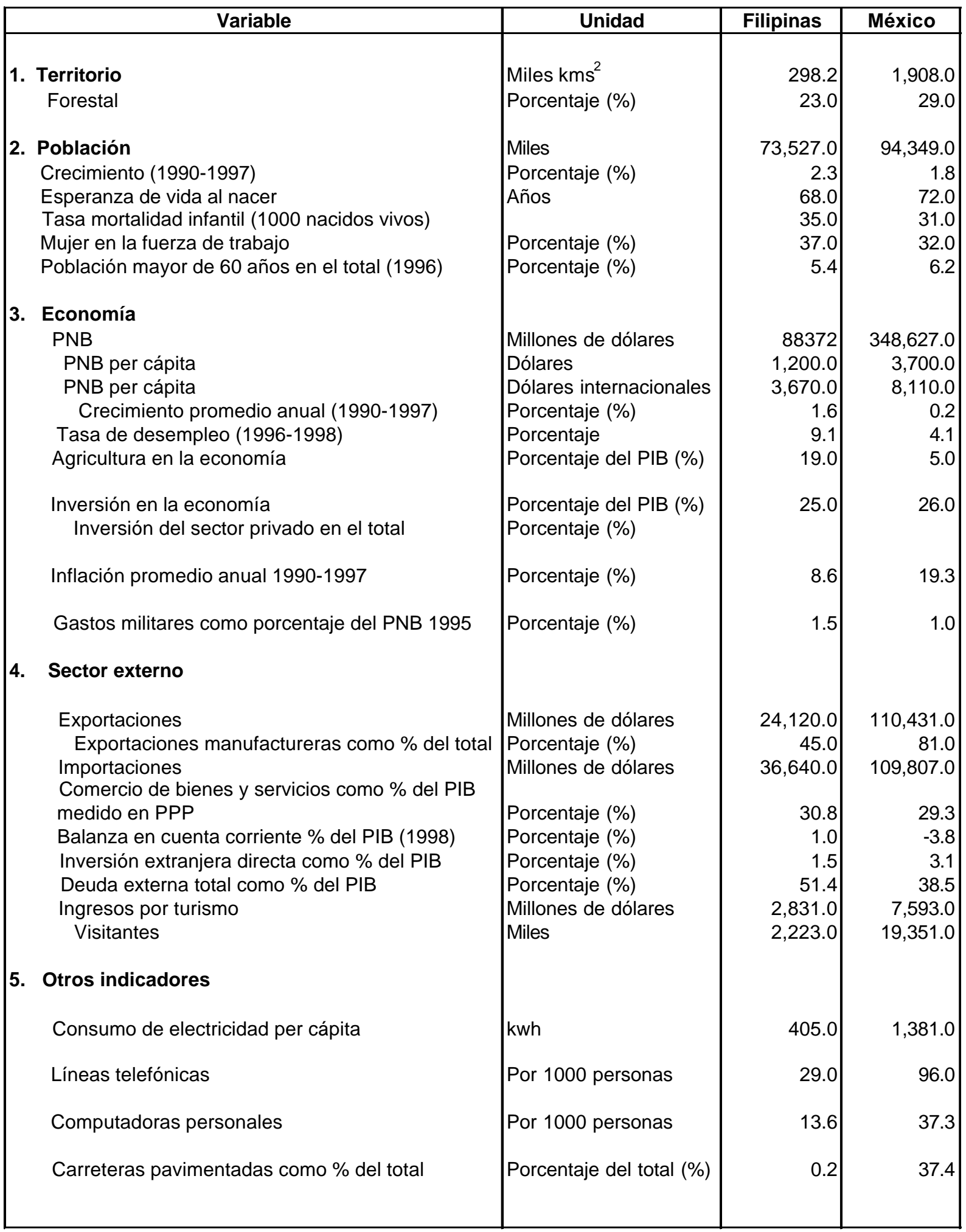

$\mathrm{PPP}=$ Paridad del poder de compra; $\mathrm{PIB}=$ Producto Interno Bruto; $\mathrm{PNB}=$ Producto Nacional Bruto

Fuente: World Bank, World Bank Atlas 1999, Informe Anual Banxico, 1999 y Stat-USA, Department of Commerce, CD. 
descansar más en el sector privado para llevar a cabo proyectos de infraestructura vitales. Para ello ha establecido un esquema de construiroperar-transferir (BOT, por sus siglas en inglés). En cuanto al sector agrícola, el gobierno ha lanzado un programa de seguridad alimentaria para impulsar el sector.

\section{Nivel de vida}

Como se apuntó anteriormente, el nivel de ingreso de los filipinos es todavía muy bajo en comparación al de sus vecinos del sudeste asiático, con excepción de Indonesia. Los habitantes de Malasia y Tailandia presentan niveles de ingreso que constituyen el doble del de los filipinos. Otros indicadores de la calidad de vida confirman lo anterior: el consumo de electricidad per cápita en Filipinas es un tercio del de los mexicanos; el número de líneas telefónicas por cada mil personas es de 29 en Filipinas y de 96 en México; el porcentaje de carreteras pavimentadas con respecto al total, es apenas de 0.2 por ciento en Filipinas, mientras en México es de 37 por ciento, y las computadoras personales por cada mil personas es de 13.6 en Filipinas y de 37 en México.

Aunque el PIB per cápita de los filipinos mostró una tasa de crecimiento promedio de uno por ciento durante el período 1969-1999, este indicador no refleja una de las paradojas: la convivencia de la pobreza con la riqueza. Efectivamente, la distribución de la riqueza en Filipinas ha permanecido muy desigual. Así, en 1975,20 por ciento de la población más pobre tenía acceso a 6 por ciento del ingreso del país, mientras que 20 por ciento de la población de mayores recursos controlaba 56 por ciento del ingreso. Para 1997, la situación no es mejor, el quintil más pobre controla 5.9 del ingreso y el más rico, 49.6 por ciento.

De acuerdo con algunos estudios, esta situación es el resultado del fuerte deterioro de los salarios reales, principal fuente de ingreso de las clases medias y bajas. ${ }^{2}$ Por un lado, el salario nominal se mantuvo bajo por mucho tiempo debido al exceso de oferta de trabajo. Esta situación, a su vez, fue el resultado de que, sobre todo cuando la economía permaneció cerrada, el lento desarrollo de la industria no permitía generar las fuentes de empleo necesarias para absorber la mano de obra que emigraba del campo. En este último, los avances tecnológicos permitieron ahorrar mano de obra y ello agravó la situación del desempleo. Así, mientras el salario nominal permanecía bajo y la inflación se mantenía por arriba de los dígitos, los salarios reales se deterioraban. En consecuencia, los altos niveles de desempleo han coexistido con elevados niveles de migración en Filipinas.

Lo paradójico de lo anterior es que el nivel de educación del pueblo filipino es relativamente alto. La matrícula en primaria es de 100 por ciento y las tres cuartas partes de los alumnos alcanzan hasta el quinto año. En secundaria la matrícula es de 60 por ciento. La participación de las mujeres en la matrícula en ambos niveles es cercana a la mitad. La participación de la mujer en la fuerza de trabajo es de 37 por ciento, cinco puntos por arriba de la mexicana. La escolaridad de los adultos es de 7 años, similar a la mexicana, y el analfabetismo entre los adultos mayores de 15 años es de 5.4 por ciento. Sin embargo, mucha de esta población con calificación, al no encontrar oportunidades de trabajo en su país, emigran a otros lugares.

En 1974 el presidente Marcos lanzó un programa de empleo en el exterior, diseñado de manera temporal para reducir los conflictos internos y al mismo tiempo tomar ventaja de los mercados globales de empleo. Internamente, se esperaba que este programa redujera las presiones del desempleo y ayudara a disminuir los problemas de balanza de pagos a través de remesas obligatorias. También se esperaba aprovechar la creciente demanda de mano de obra por parte de los nuevos ricos países petroleros. Al inicio este programa fue monopolio del gobierno, pero en 1978 se permitió la participación del sector privado en la exportación de servicios laborales. No obstante que fue concebido como un programa temporal, ahora se ha institucionalizado y está muy arraigado entre los filipinos. En Manila, alrededor de 11 por ciento de las familias tienen como principal 
fuente de ingreso las remesas del exterior. ${ }^{3}$ Recientemente, se han dado dos cambios importantes en el empleo filipino en el exterior: el mercado se ha desplazado del medio oriente a Asia Pacífico y los flujos migratorios están compuestos en su mayoría por mujeres.

\section{Consideraciones finales}

Aunque Filipinas ha sido el país del sudeste asiático que no ha mostrado el éxito obtenido por sus vecinos en el desarrollo de la economía, cuenta con los recursos humanos y materiales para avanzar hacia el nivel de un país desarrollado. Entre algunos de los problemas estructurales más importantes que debe enfrentar este país se encuentran la debilidad de las finanzas públicas, la deficiente distribución del ingreso y los bajos niveles de ahorro.

Las finanzas públicas débiles inhiben el desarrollo de una muy necesitada infraestructura y la oferta de servicios básicos, cuya escasez puede afectar negativamente la competitividad internacional de este país en un mundo globalizado. Por un lado, los ingresos del gobierno enfrentan problemas de administración y evasión, y por otro, tres cuartas partes del gasto público ya están comprometidas en el pago a la burocracia, los desembolsos por el servicio de la deuda y las transferencias a los gobiernos locales. Así, el margen de maniobra del gobierno para impulsar proyectos de inversión en infraestructura es muy limitado.

Otro de los problemas que enfrenta la economía de Filipinas es la baja tasa de ahorro de la población, lo cual está estrechamente ligado a la desigual distribución del ingreso. Por lo tanto, para financiar la inversión ha sido necesario recurrir al endeudamiento externo. No obstante, aunque la deuda externa ha seguido creciendo, el servicio de la deuda ya no impone una carga tan pesada como a principios de los ochenta. Actualmente, ésta representa 12 por ciento de las exportaciones. Lo anterior es resultado de que Filipinas ha logrado renegociar los plazos de amortización de la deuda externa y la mayor parte de ella tiene vencimiento de largo y mediano plazo.

Otro de los sectores que merece especial atención en Filipinas es el agrícola, por el peso tan importante que todavía tiene en la economía. Aumentar la productividad del sector es uno de los retos primordiales que enfrenta la presente administración.

Por último y vinculado a lo anterior, está el problema no resuelto de la desigual distribución del ingreso, excesivamente concentrado en unos pocos. La reforma fiscal, la oferta de una infraestructura y servicios básicos adecuados y la implementación de una política agraria que redistribuya el ingreso en el campo, son los desafíos que es necesario enfrentar para elevar el nivel de vida del pueblo filipino.

\section{Notas}

1 Vera Simone y Anne Thompson Feraru. Political and Economic Development in a Global Context, p. 197.

2 Richard Hooley. "Economic Developments in the Philippines" en Democracy \& Develpment in East Asia. Taiwan, South Korea, and The Philippines, Ed. Robinson, Thomas, The AEI Press, 1991, pp. 193-213.

3 James Tyner y Daniel Donaldson. "The geography of Phillipine international labour mitration fields" en Asia Pacific Viewpoint, vol. 40, no. 3, December 1999, pp. 217-235.

\section{Fuentes}

Eccleston, Bernard, Dawson, Michael y McNamara, Deborah, The Asia-Pacific Profile, Routledge, The Open University, 1998, PP. 182-187; Borthwick, Mark, Pacific Century. The Emergence of Modern Pacific Asia, The Pacific Basin Institute, 1992, pp. 342-350; Stat-USA, US Department of Commerce, CD, Varios reportes: Philippines: 1998 Country Report on Economic Policy and Trade Practices; The Philippines, Country Analysis Brief; The New Encyclopaedia Britannica, vol. 25, 15 ${ }^{\text {th }}$ Edition, 1998, pp. 537-543. Пח?:- 